\title{
The Two-Dimensional Power Spectrum of the Las Campanas Redshift Survey: Detection of Excess Power on 100 [ITAL]h[/ITAL][TSUP]-1[/TSUP] Mpc Scales
}

\section{Citation}

Landy, Stephen D., Stephen A. Shectman, Huan Lin, Robert P. Kirshner, Augustus A. Oemler, and Douglas Tucker. 1996. "The Two-Dimensional Power Spectrum of the Las Campanas Redshift Survey: Detection of Excess Power on 100 [ITAL]h[/ITAL][TSUP]-1[/TSUP] Mpc Scales." The Astrophysical Journal 456 (1). https://doi.org/10.1086/309863.

\section{Permanent link}

http://nrs.harvard.edu/urn-3:HUL.InstRepos:41399942

\section{Terms of Use}

This article was downloaded from Harvard University's DASH repository, and is made available under the terms and conditions applicable to Other Posted Material, as set forth at http:// nrs.harvard.edu/urn-3:HUL.InstRepos:dash.current.terms-of-use\#LAA

\section{Share Your Story}

The Harvard community has made this article openly available.

Please share how this access benefits you. Submit a story.

\section{Accessibility}


The Astrophysical Journal, 456:L1-L4, 1996 January 1

(c) 1996. The American Astronomical Society. All rights reserved. Printed in U.S.A.

\title{
THE TWO-DIMENSIONAL POWER SPECTRUM OF THE LAS CAMPANAS REDSHIFT SURVEY: DETECTION OF EXCESS POWER ON $100 h^{-1}$ Mpc SCALES
}

\author{
Stephen D. Landy and Stephen A. Shectman \\ Carnegie Observatories, 813 Santa Barbara Street, Pasadena, CA 91101 \\ HuAn Lin AND Robert P. KIRSHNER \\ Harvard-Smithsonian Center for Astrophysics, 60 Garden Street, Cambridge, MA 02138 \\ Augustus A. Oemler \\ Department of Astronomy, Yale University, New Haven, CT 06520-8101 \\ AND \\ DOUgLas TucKer \\ Astrophysikalisches Institut Potsdam, An der Sternwarte 16, D-14482 Potsdam, Germany \\ Received 1995 September 13; accepted 1995 October 13
}

\begin{abstract}
We have measured the two-dimensional power spectrum of the Las Campanas Redshift Survey on scales between 30 and $200 h^{-1} \mathrm{Mpc}\left(q_{0}=0.5, H_{0}=100 h \mathrm{~km} \mathrm{~s}^{-1} \mathrm{Mpc}^{-1}\right)$. Such an analysis is more sensitive to structure on scales greater than $50 \mathrm{~h}^{-1} \mathrm{Mpc}$ than a full three-dimensional analysis, given the geometry of the survey. We find a strong peak in the power spectrum at $\sim 100 h^{-1} \mathrm{Mpc}$ relative to the smooth continuum expected from the best-fit cold dark matter model (Prob $\sim 2.5 \times 10^{-4}$ with $\Omega h=0.3$ assuming a Gaussian random field). This signal is detected in two independent directions on the sky and has been identified with numerous structures visible in the survey that appear as walls and voids. Therefore, we conclude that there exists a significant increase in power on this scale and that such structures are common features in the local universe, $z \leq 0.2$.
\end{abstract}

Subject headings: cosmology: observations — galaxies: clusters: general — large-scale structure of universe

\section{INTRODUCTION}

The existence of large density fluctuations in the galaxy distribution on the order of $100 h^{-1} \mathrm{Mpc}$ in size has been known since the discovery of the Bootes void in the early $1980 \mathrm{~s}$ (Kirshner et al. 1981). Other early evidence of structure on such scales was found by Chincarini, Giovanelli, \& Haynes (1983) and de Lapparent, Geller, \& Huchra (1986). Subsequently, Geller \& Huchra (1989) reported the discovery of the "Great Wall," a large wall-like distribution of galaxies over 100 $h^{-1} \mathrm{Mpc}$ in extent. More recently, Broadhurst et al. (1990) have claimed detection of a sharp spike in the one-dimensional power spectrum around $128 h^{-1}$ Mpc using deep pencil-beam surveys (see also Mo et al. 1992).

Although it is not unexpected that large structures should exist according to currently popular working theories of largescale structure formation, such as a cold dark matter (CDM) power spectrum coupled with the "pancake" scenario of structure formation as predicted by Zeldovich, the existence of excess power on a preferred scale is problematic. However, there do exist physically motivated reasons to expect some perturbation in the power spectrum on these scales. For example, this scale is on the order of the horizon size at mass-radiation equality, and an "acoustic peak" in the power spectrum on these scales is a prediction of many standard cosmologies (see Peebles 1993, § 25).

To make a definitive measurement of galaxy density fluctuations in this regime requires a large number of independent volumes situated over a broad region of the sky. The Las Campanas Redshift Survey includes over 26,000 galaxies with a mean redshift of $z=0.1$ and is well suited for making such measurements. The survey consists of six slices, each approx- imately 1.5 thick in declination by $80^{\circ}$ wide in right ascension. In each hemisphere the slices are centered on the same right ascension while being offset in declination by $3^{\circ}$ or $6^{\circ}$. The three slices in the south Galactic hemisphere are located at $-39^{\circ},-42^{\circ}$, and $-45^{\circ}$ decl. centered on $0^{\mathrm{h}} 45^{\mathrm{m}}$ R.A., and the three in the north at $-3^{\circ},-6^{\circ}$, and $-12^{\circ}$ decl. centered on $12^{\mathrm{h}} 45^{\mathrm{m}}$ R.A. A more detailed description of the survey, including a detailed discussion of the preparation of the redshift sample and various selection effects, appears in Shectman et al. (1995) and Lin et al. (1996a).

\section{POWER SPECTRUM ANALYSIS}

In order to estimate the two-dimensional power spectrum, we principally follow the analysis outlined by Peacock \& Nicholson (1991) and Feldman, Kaiser, \& Peacock (1994) with some minor changes (see also Burbidge \& O'Dell 1972; Webster 1976; Park et al. 1994). Each slice was analyzed separately. The coordinates of each slice were rotated in order to maximize the projection of the slice in the $x-y$ plane. The data were cut to include only those galaxies with $10,000 \mathrm{~km}$ $\mathrm{s}^{-1} \leq v_{z} \leq 45,000 \mathrm{~km} \mathrm{~s}^{-1}$ in order to minimize the effects of uncertainties in the selection function. For fields in which the number of redshifts observed was less than that observable given the magnitude limits for the field, the observed points were weighted by $\left(f_{\text {obs }}\right)^{-1}$, where $f_{\text {obs }}$ is the observed fraction (see also Lin et al. 1996a). This weighting approximates uniform weighting for a magnitude-limited sample.

The galaxies were expanded in plane waves and normalized in the standard manner. The Fourier expansion is given by

$$
a_{\boldsymbol{k}} \equiv N_{\mathrm{wt}}^{-1} \sum_{i}\left(f_{\mathrm{obs} i}\right)^{-1} \exp \left(i \boldsymbol{k} \cdot \boldsymbol{x}_{i}\right)
$$


where $N_{\text {wt }}$ is the weighted number of galaxies in the sample, $f_{\text {obs }_{i}}$ is the appropriate fraction, $\boldsymbol{x}_{\boldsymbol{i}}$ is the galaxy's two-dimensional coordinates $\left(x_{i}, y_{i}\right)$, and $a_{k}=\left(a_{k x}, a_{k y}\right)$ is the complex Fourier coefficient of the two-dimensional Fourier transform at wavenumber $\boldsymbol{k}=\left(k_{x}, k_{y}\right)$. The complex Fourier coefficients $w_{k}$ of the window function with power spectrum $W_{2 \mathrm{D}}(\boldsymbol{k})$ were determined using the same angular and radial selection function as that of the data but with 50 times the number of galaxies distributed randomly. The window function is simply the Fourier transform of the selection function. The Fourier transform of the random catalog was then subtracted from that of the data and the power spectrum calculated (see Feldman et al. 1994).

Converting sums to integrals, the normalized convolved estimate of the two-dimensional power spectrum $\hat{P}_{2 \mathrm{D}}(\boldsymbol{k})$ is

$$
\hat{P}_{2 \mathrm{D}}(\boldsymbol{k}) \equiv \frac{\int d^{2} \hat{\boldsymbol{k}} W_{2 \mathrm{D}}(\boldsymbol{k}-\hat{\boldsymbol{k}})\left[P_{2 \mathrm{D}}(\dot{\boldsymbol{k}})+S_{2 \mathrm{D}}\right]}{\int d^{2} \boldsymbol{k} W_{2 \mathrm{D}}(\boldsymbol{k})} .
$$

This expression shows the well-known result that the true power spectrum $P_{2 \mathrm{D}}(\boldsymbol{k})$ plus the shot noise $S_{2 \mathrm{D}}$ is convolved with the power spectrum of the two-dimensional window function $W_{2 \mathrm{D}}(\boldsymbol{k})$ to give the measured signal $\hat{P}_{2 \mathrm{D}}(\boldsymbol{k})$. At the wavelengths reported in this Letter, the correction term due to subtraction of the DC level is negligible and will be ignored (Peacock \& Nicholson 1991, eq. [25]).

\subsection{Calculation of the Two-dimensional Power Spectrum}

The two-dimensional power spectrum $P_{2 \mathrm{D}}\left(k_{x}, k_{y}\right)$ is a projection of the three-dimensional spectrum $P_{3 \mathrm{D}}\left(k_{x}, k_{y}, k_{z}\right)$ onto the $k_{x}-k_{y}$ plane by way of a window and depends on the geometry and orientation of the survey. As a heuristic, in this development one can approximate the survey as having an effective volume with dimensions $\left(d_{x}, d_{y}, d_{z}\right)$, where $d_{z} \ll d_{x}$, $d_{y}$, and model the window functions in each of these directions as Gaussians with width $\sigma_{i} \sim \pi / d$. In such a case the window function is separable, and the relationship between the twodimensional and three-dimensional power spectrum is easily seen. Calculations for a one-dimensional projection and further discussion can be found in Szalay et al. (1991).

In a full three-dimensional analysis neglecting the shot noise, the power spectrum estimator is

$$
\begin{aligned}
& \hat{P}_{3 \mathrm{D}}\left(k_{x}, k_{y}, k_{z}\right)= \frac{1}{(2 \pi)^{3 / 2} \sigma_{x} \sigma_{y} \sigma_{z}} \\
& \times \iiint_{-\infty}^{\infty} d \hat{k}_{x} d \dot{k}_{y} d \dot{k}_{z} P_{3 D}\left(\hat{k}_{x}, \hat{k}_{y}, \hat{k}_{z}\right) \\
& \times e^{-\left[\left(k_{x}-\dot{k}_{x}\right)^{2} / 2 \sigma_{x}^{2}\right]} e^{-\left[\left(k_{y}-\hat{k}_{y}\right)^{2} / 2 \sigma_{y}^{2}\right]} e^{\left.-\left[k_{z}-\dot{k}_{z}\right)^{2} / 2 \sigma^{2}\right]} .
\end{aligned}
$$

In our two-dimensional analysis, the volume is collapsed along the $z$-direction, which results in a planar survey with dimensions $\left(d_{x}, d_{y}\right)$ and a projection of power onto the $k_{x}-k_{y}$ plane. It can be shown that the resulting two-dimensional spectrum is given by the $k_{z}=0$ component of the fully convolved three-dimensional power spectrum. Therefore, we may define

$$
P_{2 \mathrm{D}}\left(\hat{k}_{x}, \hat{k}_{y}\right) \equiv \frac{1}{\sqrt{2 \pi} \sigma_{z}} \int_{-\infty}^{\infty} d \hat{k}_{z} P_{3 \mathrm{D}}\left(\hat{k}_{x}, \hat{k}_{y}, \hat{k}_{z}\right) e^{-\left(k_{z}^{2} / 2 \sigma_{z}^{2}\right)},
$$

which formally illustrates the projection. Then

$$
\begin{aligned}
& \hat{P}_{2 \mathrm{D}}\left(k_{x}, k_{y}\right)=\frac{1}{2 \pi \sigma_{x} \sigma_{y}} \\
& \quad \times \iint_{-\infty}^{\infty} d \hat{k}_{x} d \hat{k}_{y} P_{2 \mathrm{D}}\left(\hat{k}_{x}, \hat{k}_{y}\right) e^{-\left[\left(k_{x}-\hat{k}_{x}\right)^{2} / 2 \sigma x^{2}\right]} e^{-\left[\left(k_{y}-\hat{k}_{y}\right)^{2} / 2 \sigma_{y}\right]},
\end{aligned}
$$

for consistency with equation (2). Note that our definition of the two-dimensional spectrum includes the normalization factor $\left(\sqrt{2 \pi} \sigma_{z}\right)^{-1}$ for direct comparison with the $\hat{P}_{3 \mathrm{D}}\left(k_{x}, k_{y}, k_{z}=0\right)$ component of the full three-dimensional spectrum. Therefore, our results are reported in units of $\left(h^{-1} \mathrm{Mpc}\right)^{3}$. For all subsequent analysis, the exact window functions have been calculated numerically, and the Gaussian approximation has not been made.

\section{RESULTS}

\subsection{CDM Simulations}

In order to check our results and investigate the effects of the survey's geometry, selection functions, and those due to redshift distortions, we performed an identical analysis on synthetic data kindly supplied by Changbom Park (see Park et al. 1994). Fifty-six realizations of sets of the three southern slices were culled from a large CDM simulation, $\left(576 h^{-1}\right.$ $\mathrm{Mpc})^{3}$ with $\Omega h=0.2$, using the same selection function and geometry as the survey's. The two-dimensional power spectrum for each slice in a set was calculated and then averaged together to generate a mean power spectrum for each set. The mean result for all 56 sets is shown in Figure $1 b$ together with error bars derived from these data. For all slices, the shotnoise power was less than $250\left(h^{-1} \mathrm{Mpc}\right)^{3}$.

Also shown in Figure $1 b$ is a line indicating the expected two-dimensional power spectrum after projection and convolution of the underlying three-dimensional power spectrum of the simulation. The window functions for each slice were calculated numerically and then used to project and convolve the analytic three-dimensional spectrum. As may be seen, there is excellent agreement between the synthetic data and the expected analytic result. This indicates that effects due to wavelength-dependent redshift distortions are expected to be minimal in this regime.

Also plotted is the envelope of $1 \sigma$ fluctuations based on the amplitude of the expected signal and the degrees of freedom calculated from the number of independent modes for a given wavenumber $|\boldsymbol{k}|$ given the effective area of the two-dimensional window function (FWHM: $2.20 \times 10^{-4} h^{-2} \mathrm{Mpc}^{2}$ ). The good agreement shows that a formal analysis based on a model spectrum, calculated degrees of freedom, and Gaussian random field is highly accurate. A similar technique will by necessity be used with the survey data.

\subsection{Las Campanas Survey Results}

The mean result for all six survey slices is shown in Figure $1 a$. Figure 2 shows the individual measured power spectrum for each slice and the mean north and south signals. Since for the survey data it is not possible to know the underlying three-dimensional power spectrum a priori, we calculated the expected power spectrum given a class of linear CDM models with $0.2 \leq \Omega h \leq 0.5$ (Efstathiou, Bond, \& White 1992; Bond $\&$ Efstathiou 1984). These models were projected and convolved as above and fitted to the measured signal between 30 $h^{-1} \mathrm{Mpc}$ and $70 h^{-1} \mathrm{Mpc}$ using only their overall amplitude as a free parameter. The best-fit spectrum was given by $\Omega h=0.3$ and is shown in Figure $1 a$. 


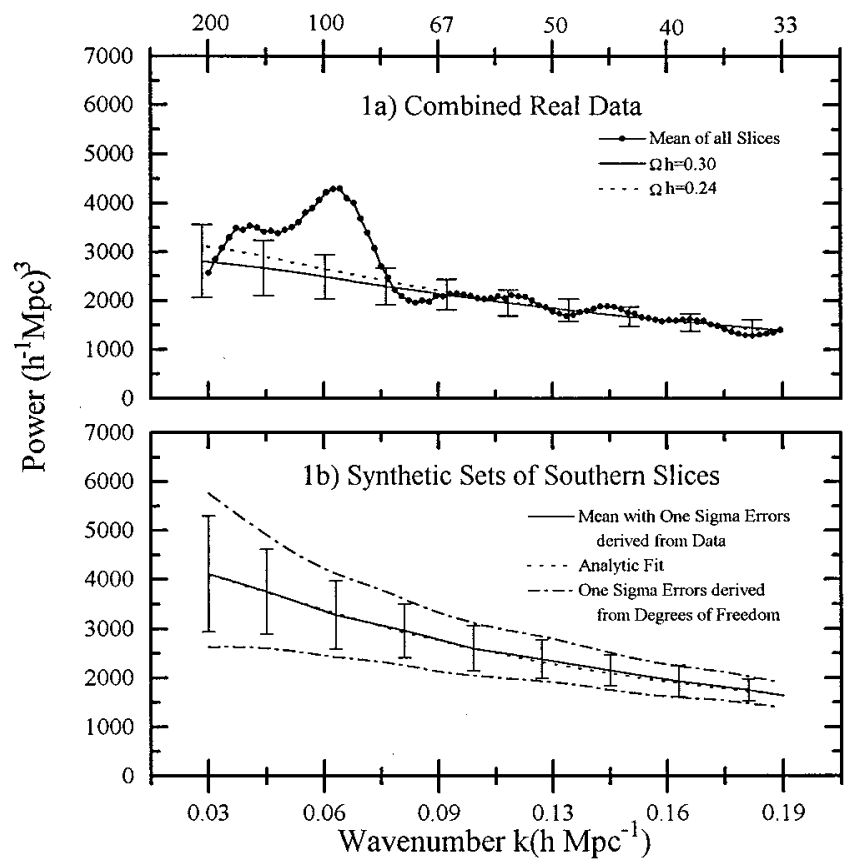

FIG. 1.-Comparison of the average signal from all six slices of real data compared to 56 sets of synthetic data with the same geometry and selection function as the three southern slices $\left(-39^{\circ},-42^{\circ}\right.$, and $-45^{\circ}$ decl.). For the synthetic data, the $1 \sigma$ error bars are derived from the sets themselves. The 1 $\sigma$ error envelope is based on the calculated degrees of freedom at each wavelength together with the mean signal. The analytic spectrum is calculated using the underlying power spectrum in the synthetic data projected and convolved with the window functions. For the real data, a best-fit power spectrum was derived by projecting and convolving linear CDM power spectra with $\Omega h$ between 0.2 and 0.5 and fitting the result to the real data between 30 and $70 h^{-1} \mathrm{Mpc}$. The best fit was found to be $\Omega h=0.3$ and is shown in the graph. The error bars are calculated using the expected signal together with degree of freedom analysis. Also plotted is the best-fit spectrum with $\Omega h=0.24$. This is shown for comparison with the findings of the one-in-six QDOT IRAS survey.

As is evident, the measured signal below $70 h^{-1} \mathrm{Mpc}$ and the fit are in good agreement; however, a strong excess of power appears at $\sim 100 h^{-1}$ Mpc. This peak is essentially unresolved since its width is approximately that expected solely from convolution with the window function. The error bars are derived from analysis of the degrees of freedom at each wavenumber. Significant excess power at wavelengths at or above $100 \mathrm{~h}^{-1} \mathrm{Mpc}$ is evident in five of the six slices in Figure 2.

To be conservative in our analysis of the significance of this peak, we consider each set of three slices as one independent sample. The mean power spectrum at this wavenumber, $|\boldsymbol{k}|=0.067$, consists of an average over approximately 15 independent modes in the north and 15 in the south with 2 degrees of freedom in each mode giving a total of 60 degrees of freedom. The amplitude of the measured peak at this point is approximately 1.76 times the expected signal as given by the $\Omega h=0.3$ model. Taking the null hypothesis that the local universe on these scales is drawn from a Gaussian random field (as reported by Feldman et al. 1994), the significance of this peak is $\sim 2.5 \times 10^{-4}$, that is, a peak of this amplitude at this wavenumber would be expected by chance once in $\sim 3900$ similar surveys. Also shown for comparison is the best fit for an $\Omega h=0.24$ model, which was the result found for the updated one-in-six QDOT IRAS survey (Feldman et al. 1994). In this case, the significance is $\sim 1.1 \times 10^{-3}$.
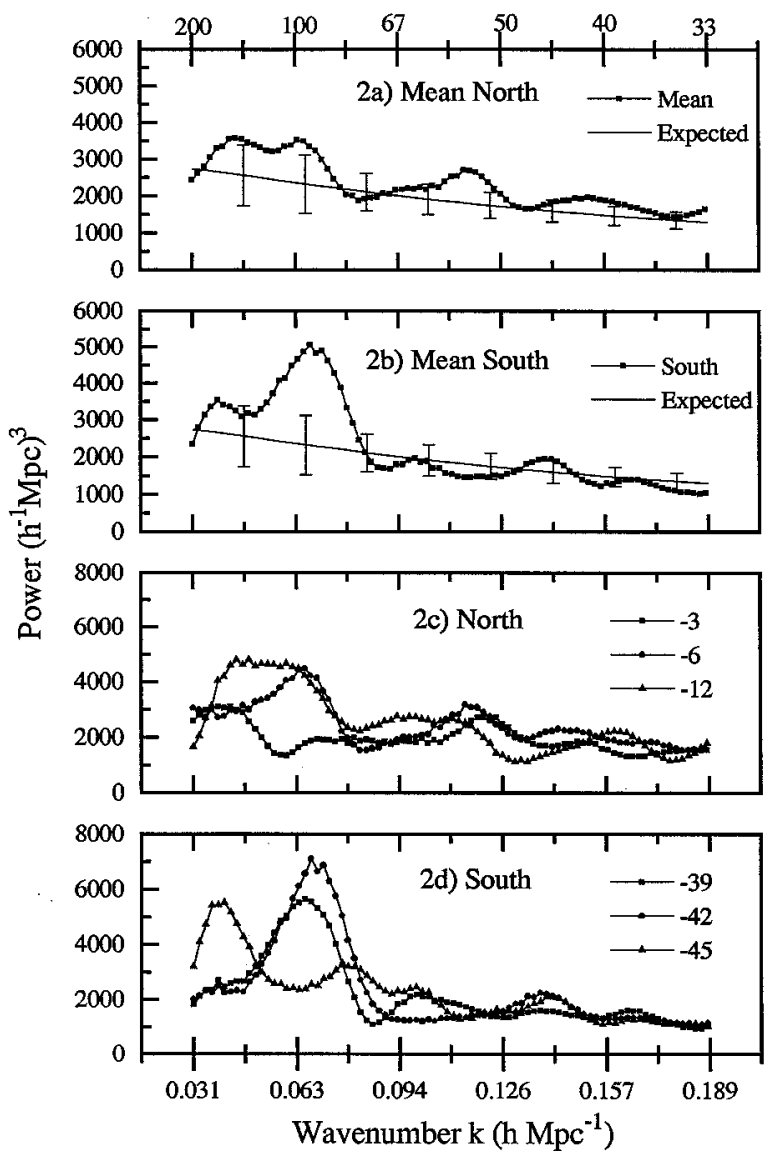

FIG. 2.-Individual graphs showing the signals from each of the six slices together with the mean signals for the north $\left(-3^{\circ},-6^{\circ}\right.$, and $-12^{\circ} \mathrm{decl}$.) and south $\left(-39^{\circ},-42^{\circ}\right.$, and $-45^{\circ}$ decl.) sets. Both directions show significant excess power at $\sim 100 h^{-1} \mathrm{Mpc}$. Peaks above $100 \mathrm{~h}^{-1} \mathrm{Mpc}$ are evident in five of the six slices. One sigma error bars are shown for only the north and south means as the individual slices in each hemisphere are not independent. For all slices, the shot-noise power was less than $250\left(h^{-1} \mathrm{Mpc}\right)^{3}$.

Amendola (1994) has shown that the significance of a peak may be overestimated given the null hypothesis of a Gaussian random field because of the higher order correlations in the density field, albeit the simulations indicate that this effect is not important at these wavenumbers (Fig. 1b). The significance may also be modified by considering that one is also able to measure peaks at other wavenumbers. However, the existence of a peak on these scales has been anticipated by Broadhurst et al. (1990) using one-dimensional pencil-beam surveys.

\section{DISCUSSION AND ANALYSIS}

\subsection{Comparison to a Three-dimensional Analysis and Other Surveys}

A question is, what are the advantages of a two-dimensional versus a three-dimensional analysis (see Lin et al. 1996b) at these wavenumbers? This is answered by comparing the differences between a one-dimensional projection and twodimensional convolution versus a straight three-dimensional convolution given the geometry of the survey. To first order, the effective dimensions of the survey given a full set of three slices in one hemisphere are $300 \times 400 \times 50 \mathrm{~h}^{-1} \mathrm{Mpc}$. In the geometry of the two-dimensional analysis, the survey dimensions are $300 \times 400 h^{-1} \mathrm{Mpc}$. This affords several significant 
advantages, the most obvious being that a two-dimensional analysis can fairly sample wavelengths up to several hundred $h^{-1} \mathrm{Mpc}$. On the other hand, a full three-dimensional analysis samples wavevectors at all orientations to the planes of the slices. As a result, the measured signal becomes dominated by aliased power at wavelengths above $\sim 50 h^{-1} \mathrm{Mpc}$, the effective thickness of a set of slices.

A well-sampled two-dimensional analysis also has advantages as regards the detection of non-Gaussian structures since such structures show up as strong, localized peaks at a specific wavevector in the power spectrum. Depending on how the survey has cut through such structures, these peaks may show up at their true frequency or be projected to other wavelengths. However, the excess power will still show up as a strong, local peak and be easily detected in a two-dimensional analysis. In a three-dimensional analysis in this geometry, such peaks would be smoothed over many directions and wavelengths because of the large width of the window function in the third dimension, which makes such sharp features more difficult to detect.

Other surveys, such as the CfA2 (Geller \& Huchra 1989; Vogeley et al. 1992) and SSRS2 (da Costa et al. 1994a), although sampling structure at higher densities, are limited at these wavelengths. Recent work by da Costa et al. (1994b) reports analysis of volumes with a depth of $\sim 130 h^{-1} \mathrm{Mpc}$. As these surveys contain only a few independent modes at this scale, it is not surprising that they might not detect such a peak. This signal has also not been detected in recent analyses of the IRAS survey (see Fisher et al. 1993; Feldman et al. 1994). The latter analysis has on the order of the same effective volume as ours; however, the sampling density is a factor of 10-15 less, and the shot noise is on the order of the signal. In our survey, the sampling density and signal-to-noise ratio is a factor of 10 greater. Also, it has been shown that the detection of structure is a function of sampling density and signal-to-noise ratio (see Szapudi \& Szalay 1996).

\subsection{Identification of Contributing Structures in the Survey}

In order to determine what structures are responsible for these spikes, maxima of the plane waves corresponding to the largest peaks above $\sim 100 h^{-1} \mathrm{Mpc}$ have been overlaid on maps of the real space distribution of galaxies for a slice from each hemisphere, $-12^{\circ}$ and $-39^{\circ}$. The appropriate peaks have been identified as well on the conjugate two-dimensional power spectrum contour maps of the same slices. These are shown in Figure 3 (Plates L1-L2). The maxima of the plane waves, with phases from the Fourier transform, have been plotted as straight lines in Figure 3. Large structures with the appearance of walls and voids being traced out by superclusters are being detected by the two-dimensional power spectrum analysis, which gives strong visual confirmation of the results.

It is not unexpected that the strongest peak on Figure 3 corresponds to a wavevector pointing in the redshift direction as this may be due to amplitude enhancements from redshift distortions (see Kaiser 1987). However, the existence of numerous other peaks and structures with different orientations at this same scale precludes interpretations based principally upon this effect.

\subsection{Gaussian or Non-Gaussian Structures}

Of interest to the theory of structure formation is whether this excess power is the result of the existence of non-Gaussian structures or is rather an inherent increase in power. Unfortunately, power spectrum statistics are not that robust in making such a determination (see Amendola 1994). An analysis based upon the distribution of power spectrum amplitudes (see Szalay et al. 1991) has proved inconclusive, primarily because of the degree of smoothing from the window function. On the other hand, the appearance of the structures identified by the peaks is striking as is the partial coherence between the peaks as can be seen in Figure 3. Such coherence would not be expected in the case of a Gaussian random field.

\section{CONCLUSIONS}

We have detected multiple structures in the galaxy distribution in two independent regions on the sky that correspond to a peak in the two-dimensional power spectrum on the order of $100 h^{-1} \mathrm{Mpc}$, a wavelength similar to that reported by Broadhurst et al. (1990). The survey contains approximately 30 independent modes on this scale. The probability of detecting such a signal by chance, with the assumptions of a Gaussian random field and the best-fit linear CDM power spectrum, is $2.5 \times 10^{-4}\left(\Omega h=0.3\right.$ fit between $30 h^{-1} \mathrm{Mpc}$ and $\left.70 h^{-1} \mathrm{Mpc}\right)$. The structures responsible for this signal have been identified and have the appearance of walls and voids. Therefore, such large structures appear to be common features of the local universe.

The authors would like to thank Anand Sivaramakrishnan and the referee, Michael Vogeley, for useful discussions and comments. We would especially like to thank Alexander Szalay for many productive insights into the analysis and understanding of this problem. We acknowledge principal support from NSF grant AST9220460.

\section{REFERENCES}

Amendola, L. 1994, ApJ, 430, L9

Bond, J. R., \& Estathiou, G. 1984, ApJ, 285, L45

Broadhurst, T. J., Ellis, R. S., Koo, D. C., \& Szalay, A. S. 1990, Nature, 343, 726 Burbidge, G. R., \& O'Dell, S. L. 1972, ApJ, 178, 583

Chincarini, G. L., Giovanelli, R., \& Haynes, M. P. 1983, A\&A, 121, 5

da Costa, L. N., Vogeley, M. S., Geller, M. J., Huchra, J. P., \& Park, C. 1994a, ApJ, 437, L1

da Costa, L. N., et al. 1994b, ApJ, 424, L1

de Lapparent, V., Geller, M. J., \& Huchra, J. P. 1986, ApJ, 301, L1

Efstathiou, G., Bond, J. R., \& White, S. D. M. 1992, MNRAS, 258, 1

Feldman, H. A., Kaiser, N., \& Peacock, J. P. 1994, ApJ, 426, 23

Fisher, K. B., Davis, M., Strauss, M. A., Yahil, A., \& Huchra, J. P. 1993, ApJ, 402, 42

Geller, M. J., \& Huchra, J. P. 1989, Science, 246, 897

Kaiser, N. 1987, MNRAS, 227, 1

Kirshner, R. P., Oemler, A., Schechter, P. L., \& Shectman, S. A. 1981, ApJ, 248, L57

Lin, H., Kirshner, R. P., Shectman, S. A., Landy, S. D., Oemler, A., Tucker,

D. L., \& Schechter, P. L. 1996a, ApJ, submitted
Lin, H., Kirshner, R. P., Shectman, S. A., Landy, S. D., Oemler, A., Tucker, D. L., \& Schechter, P. L. 1996b, ApJ, submitted

Mo, H. J., Deng, Z. G., Xia, X. Y., Schiller, P., \& Boerner, G. 1992, A\&A, 257, 1

Park, C., Vogeley, M. S., Geller, M. J., \& Huchra, J. P. 1994, ApJ, 431, 539

Peacock, J. A., \& Nicholson, D. 1991, MNRAS, 253, 307

Peebles, P. J. E. 1993, Principles of Physical Cosmology (Princeton: Princeton Univ. Press)

Shectman, S. A., Landy, S. D., Oemler, A., Tucker, D. L., Kirshner, R. P., Lin, H., \& Schechter, P. L. 1995, in Wide-Field Spectroscopy and the Distant Universe, Proc. 35th Herstmonceux Conference, ed. S. J. Maddox \& A. Aragń-Salamanca (Singapore: World Scientific), 98

Szalay, A. S., Ellis, R. S., Koo, D. C., \& Broadhurst, T. J. 1991, in AIP Conf Proc. 222, After the First Three Minutes, ed. S. S. Holt, C. L. Bennett, \& V. Trimble (New York: AIP), 261

Szapudi, I., \& Szalay, A. S. 1996, ApJ, in press

Vogeley, M. S., Park, C., Geller, M. J., \& Huchra, J. P. 1992, ApJ, 391, L5

Webster, A. 1976, MNRAS, 175, 61 
PLATE L1
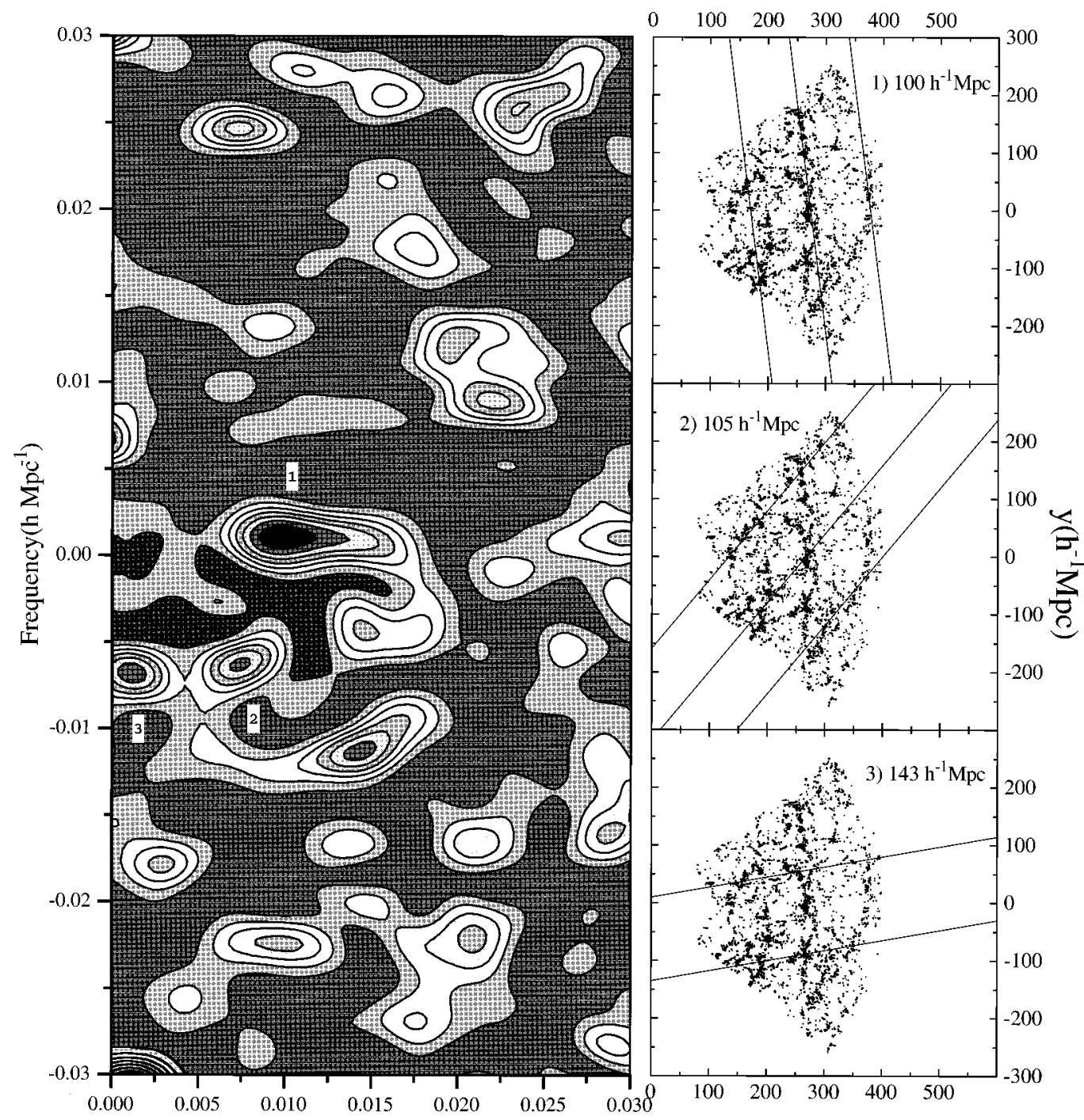

Frequency $\left(\mathrm{h} \mathrm{Mpc}^{-1}\right)$

$$
\mathrm{x}\left(\mathrm{h}^{-1} \mathrm{Mpc}\right)
$$

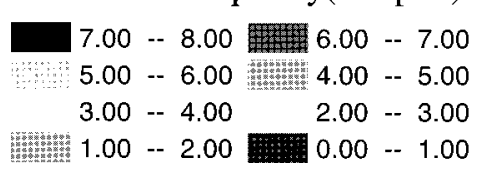

FIG. $3 a$

FIG. 3.-These plates show the two-dimensional power spectrum for the $(a)-12^{\circ}$ and $(b)-39^{\circ}$ decl. slices in the north and south, respectively. The power spectra have been normalized to the expected $\Omega h=0.3$ spectrum as a function of wavelength in order that fluctuations have the same expectation at all wavelengths. A signal of 6.1 corresponds to the expectation of only one peak of greater height over the entire graph. The width of the peaks in this figure gives a rough idea of the size of an independent mode. The coordinate of each peak $\left(k_{x}, k_{y}\right)$ in the power spectrum corresponds to the normal of a plane wave in real space. The three highest peaks above $100 h^{-1} \mathrm{Mpc}$ have been numbered and correspond to the graphs showing the real space distribution of galaxies. The straight lines indicate the maxima of these plane waves with the appropriate phase and noted wavelength. In this way it is easy to see which structures in real space are responsible for these peaks; however, true plane wave density fluctuations are not to be expected.

LANDY et al. (see 456, L4) 


\section{PLATE L2}

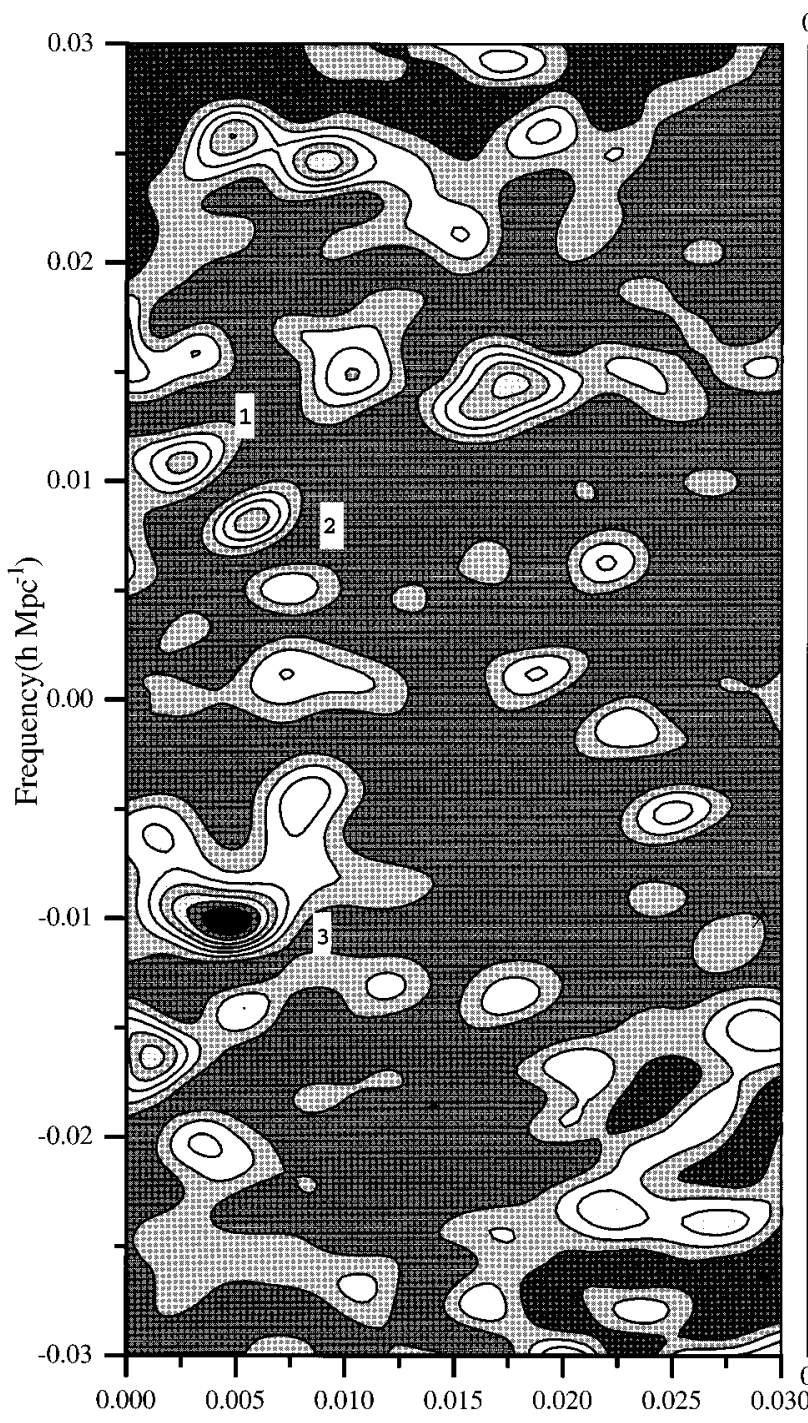

Frequency $\left(\mathrm{h} \mathrm{Mpc}^{-1}\right)$

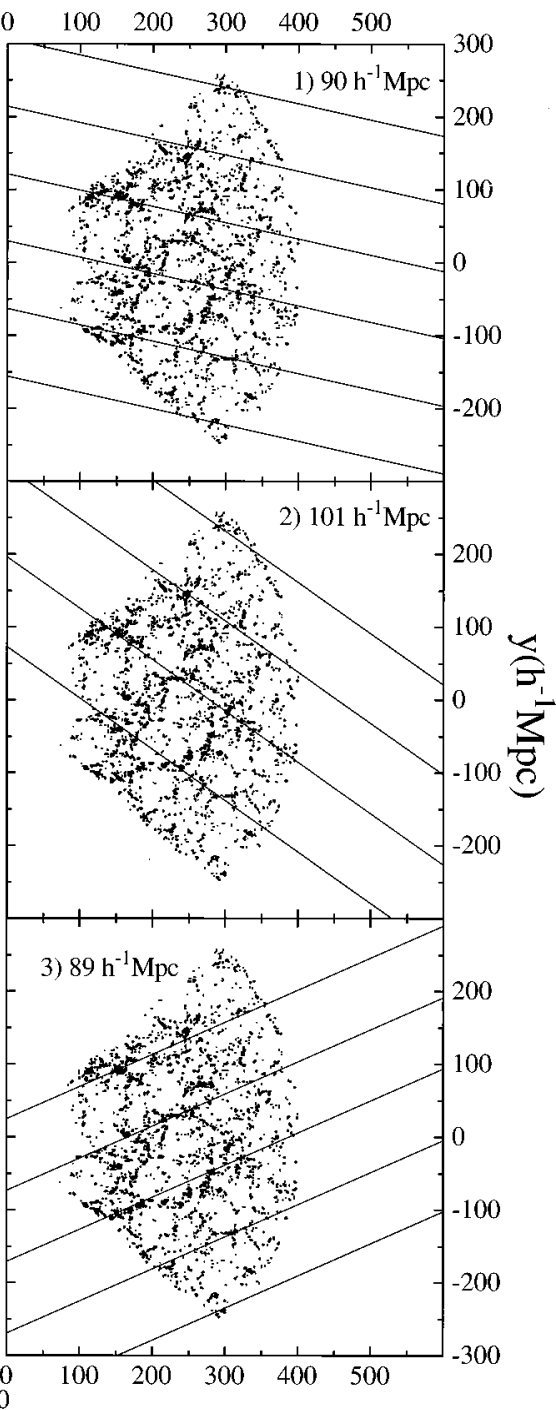

$$
\mathrm{x}\left(\mathrm{h}^{-1} \mathrm{Mpc}\right)
$$

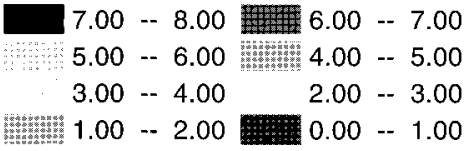

FIG. $3 b$

LANDY et al. (see 456, L4) 